\title{
The Effects of Dephosphorylation on the Structure of the Projections of Neurofilament
}

\author{
Shin-ichi Hisanaga and Nobutaka Hirokawa \\ Department of Anatomy and Cell Biology, School of Medicine, University of Tokyo, Tokyo 113, Japan
}

\begin{abstract}
Carboxy-terminal tail domains of larger molecular mass subunits (NF-M and NF-H) of neurofilaments (NFs), which are the highly phosphorylated moieties, were observed as thin flexible filaments projecting from NF core filaments by rotary shadowing (Hisanaga and Hirokawa, 1988). Dephosphorylation of NFs has been suspected to affect the structures and the functions of the carboxy-terminal tail projections. We report here the effects of the dephosphorylation on the structure of NFs studied by electron microscopy. (1) The structures of carboxy-terminal tail projections after dephosphorylation were compared with those of the control NFs by low-angle rotary shadowing. This was examined with 2 samples; the isolated neurofilaments and the short filaments assembled from NF-H. Both the dephosphorylated NFs and the short filaments showed many projections laterally extending from core filaments similar to those observed in the control samples. (2) With respect to the structure of NF in physiological solution, the density of NFs in the precipitates was examined by thin-section electron microscopy. No ditference in the density was noted between control and dephosphorylated NFs. (3) The ability to form cross-bridges in vitro was examined by quick-freeze, deep-etch electron microscopy. The structure and frequency of cross-bridges appeared to be similar in both control and dephosphorylated NFs. (4) Phosphate determination revealed that about $90 \%$ of the phosphate groups of NF-H subunit were removed by treatment with $E$. coli alkaline phosphatase. These results indicated that the dephosphorylation of NF did not affect the structure and the ability to form cross-bridges of the carboxy-terminal tail projections in vitro.
\end{abstract}

Neurofilaments (NFs) are one of the major cytoskeletal components in neuronal cells. NFs are usually composed of triplet polypeptides with different electrophoretic mobilities on SDSPAGE. In the order of their molecular mass, they are designated NF-H, NF-M, and NF-L, respectively. All subunits are divided into 3 domains: the amino-terminal head domain, the $\alpha$-helix-

\footnotetext{
Received Apr. 25, 1988; revised Aug. 16, 1988; accepted Aug. 17, 1988.

We thank Drs. J. Pudles, Y. Minami, H. Yorifuji, R. Takemura, and Y. Shiomura for their valuable suggestions and discussions for the preparation of the manuscript. We are grateful to Miss Y. Masuda for setting up the image processor to calculate the length of the filaments and the projections. This work was supported by a Special Grant-in-aid for Scientific Research No. 62065007 from the Japan Ministry of Education, Science and Culture, and a grant from the Muscular Dystrophy Association of America to N.H.

Correspondence should be addressed to Shin-ichi Hisanaga, Department of Anatomy and Cell Biology, School of Medicine, Iniversity of Tokyo, Hongo, Bunkyo-ku, Tokyo 113, Japan.
}

Copyright (c) 1989 Society for Neuroscience $0270-6474 / 89 / 030959-08 \$ 02.00 / 0$ rich rod domain, and the carboxy-terminal tail domain (Geisler et al., 1983, 1985). The $\alpha$-helix-richrod domain is responsible for the formation of the filaments. The carboxy-terminal tail domain has been suggested to constitute peripheral domains (Chin et al., 1983; Geisler et al., 1983, 1985; Julien and Mushynski, 1983). The difference in the molecular mass is due to the differences in the size of the carboxy-terminal tail domains. The carboxy-terminal tail domains of the higher-molecular-mass subunits (NF-H and NF-M) are suggested to be sites where NFs interact with each other and with other cytoplasmic organelles. We have recently demonstrated their structures both in vivo and in vitro by electron microscopy. It has been shown by the quickfreeze, deep-etch technique (QF-DE) that the NFs are extensively linked with each other by cross-bridges in the axon (Hirokawa, 1982). Immunocytochemical studies have revealed that several antibodies against the carboxy-terminal tail domains of NF-H and NF-M bind to peripheral regions of NFs (Willard and Simon, 1981; Sharp et al., 1982; Liem et al., 1985) and cross-bridges (Hirokawa ct al., 1984). In a previous paper, we showed the structure of the carboxy-terminal tail domains of the isolated NFs by low-angle rotary shadowing (Hisanaga and Hirokawa, 1988). They were thin, flexible filamentous structures about $80 \mathrm{~nm}$ long and 3-4 $\mathrm{nm}$ wide, and projected from the core filaments at an interval of $22 \mathrm{~nm}$.

The carboxy-terminal tail domains of NF-H and NF-M contain many phosphorylation sites (Jones and Williams, 1982; Julien and Mushynski, 1982, 1983; Carden et al., 1985). Major phosphorylation sites are shown to be located in a repetitive sequence motif of lysine-serine-proline (Geisler et al., 1987). Although there are some disagreements in the number of phosphorylation sites among reports, about $20-30$ phosphorylation sites in NF-H and about 10 in NF-M are indicated. Phosphorylation of NF proteins is expected to play a crucial role in the function of the NF. NFs localize differently in neuronal cells depending on the extent of their phosphorylation (Sternberger and Sternberger, 1983). The removal of phosphate from the NF-M results in the dissociation of this protein from the core filament (Wong et al., 1984). Dephosphorylation suppresses the activity of NF-H to promote microtubule polymerization (Minami and Sakai, 1985).

Since the discovery and first use by Sternberger and Sternberger (1983) of monoclonal antibodies that recognize phosphorylated and unphosphorylated epitopes differentially, many reports have indicated that NFs present in the axon are highly phosphorylated, while those in the cell bodies and the dendrites are not (Bennett and Dilullo, 1985; Peng et al., 1986; Cohen et al., 1987; Hart et al., 1987; Lee et al., 1987; Oblinger, 1987). On the other hand, QF-DE electron microscopy indicates a 
different organization of NFs in axons, on the one hand, and cell bodies/dendrites, on the other (Hirokawa, 1982). NFs in the axon run parallel and are cross-bridged extensively. Those in the cell body tend to be randomly oriented, and the number of cross-bridges are reduced to about two-fifths of the axon. Those in the dendrite run parallel but are dispersed or exist as small fascicles, and the average frequency of cross-bridges is about three-fifths of the axon, although the frequency within a dendritic fascicle is similar to that in the axon.

On the basis of these findings, we thought that phosphorylation of NF subunits alters the conformation of the projections so as to form the cross-bridges, as suggested by several recent papers (Glicksman et al., 1987; Myers et al., 1987). Using lowangle rotary shadowing and QF-DE, we have been able to probe the effects of dephosphorylation on the structure of the projections. We found that the dephosphorylated NFs have similar structures of the projections to the phosphorylated NF's and that the cross-bridges are formed between the dephosphorylated NFs as well as the control NFs. We conclude from these studies that dephosphorylation affects neither the structure of the projections nor the ability to form the cross-bridges in vitro.

\section{Materials and Methods}

Preparation of the control and the dephosphorylated NFs. NFs were prepared from bovine spinal cords by gel filtration as described in the previous paper (Hisanaga and Hirokawa, 1988). Bovine spinal cords were homogenized in an equal volume of PEM [0.1 M PIPES ( $\mathrm{pH} 6.8$ ), $1 \mathrm{~mm}$ EGTA, $1 \mathrm{~mm} \mathrm{MgCl}_{2}, 0.5 \mathrm{~mm}$ dithiothreitol, $10 \mu \mathrm{g} / \mathrm{ml}$ leupeptin, and $0.5 \mathrm{~mm}$ phenylmethylsulfonyfluoride (PMSF)]. The homogenate was centrifuged at $55,000 \times g$ for $50 \mathrm{~min}$, and the supernatant was dialyzed extensively against $50 \mathrm{~mm}$ Tris- $\mathrm{HCl}(\mathrm{pH} 8.5), 150 \mathrm{~mm} \mathrm{NaCl}$, $2 \mathrm{mM} \mathrm{MgCl}_{2}, 0.5 \mathrm{~mm}$ EGTA, $0.1 \mathrm{~mm}$ DTT, and protease inhibitors (0.1 $\mu \mathrm{g} / \mathrm{ml}$ leupeptin and $0.1 \mathrm{~mm}$ PMSF). Dephosphorylation was carried out by incubating $0.8 \mathrm{ml}$ of the dialyzed crude extract $(\sim 10 \mathrm{mg} / \mathrm{ml})$ with 40-50 units of $E$. coli alkaline phosphatase (Wako, Osaka, Japan) for $5 \mathrm{hr}$ at $35^{\circ} \mathrm{C}$. The control was treated similarly without enzyme. At the end of the incubation, the control and the dephosphorylated NFs were applied on a Sepharose CL-4B gel filtration column $(1.5 \times 27 \mathrm{~cm})$ and eluted with $\mathrm{P}_{20} \mathrm{EM}$ (20 mM PIPES, $0.5 \mathrm{~mm}$ EGTA, $1 \mathrm{~mm} \mathrm{MgCl}_{2}$, $0.1 \mathrm{~mm}$ DTT, and protease inhibitors) containing $50 \mathrm{~mm} \mathrm{NaCl}$. The fractions eluted at a void volume were used as the isolated NFs.

Purification, reassembly, and dephosphorylation of $N F-H$. NF-H was purified by the method of Geisler and Weber (1981) with slight modifications (Hisanaga and Hirokawa, 1988). Porcine spinal cords were homogenized in PEM. The homogenate was centrifuged at 55,000 $\times g$ for 50 min at $2^{\circ} \mathrm{C}$. The supernatant was made $20 \%$ with respect to glycerol and incubated for $30 \mathrm{~min}$ at $35^{\circ} \mathrm{C}$. NFs pelleted by centrifugation at $150,000 \times \mathrm{g}$ for $90 \mathrm{~min}$ were dissolved in $10 \mathrm{~mm}$ sodium phosphate (pH 7.5), $1 \mathrm{~mm}$ EGTA, $6 \mathrm{~m}$ urea, $0.5 \mathrm{~mm}$ DI\%, $0.1 \mu \mathrm{g} / \mathrm{ml}$ leupeptin, and $0.1 \mathrm{~mm}$ PMSF (buffer A). After centrifugation at $48,000 \times g$ for $30 \mathrm{~min}$, the protein solution was applied to a DEAE-cellulose column (Whatman DE-52) equilibrated with buffer A. Adsorbed proteins were eluted with a $\mathrm{NaCl}$ gradient $(0-0.25 \mathrm{M})$ in buffer $\mathrm{A}$. The fractions containing NF-H were collected and concentrated with centriflo CF-25 (W. R. Grace \& Co. Amicon division, Damvers, USA) to a protein concentration of about $2 \mathrm{mg} / \mathrm{ml}$. Concentrated NF-H $(2 \mathrm{mg} / \mathrm{ml})$ was polymerized by dialysis against $P_{20} E M$ containing $0.15 \mathrm{M} \mathrm{NaCl}$ for $3 \mathrm{hr}$ at $35^{\circ} \mathrm{C}$. After further dialysis against $50 \mathrm{~mm}$ Tris- $\mathrm{HCl}(\mathrm{pH} 8.5), 0.15$ M NaCl, $2 \mathrm{~mm} \mathrm{MgCl}_{2}, 0.5 \mathrm{~mm}$ EGTA, 0.1 mM DTT, and protease inhibitors, dephosphorylation was carried out by incubating $0.2 \mathrm{ml}$ of reassembled NF-H with 20 units of alkaline phosphatase at $35^{\circ} \mathrm{C}$ for 5 hr. The control sample was similarly treated without the enzyme. At the end of the incubation, the samples were applied on a Sepharose CL$4 \mathrm{~B}$ column $(1 \times 25 \mathrm{~cm})$ equilibrated with $\mathrm{P}_{20} \mathrm{EM}$ containing $50 \mathrm{~mm}$ $\mathrm{NaCl}$. Reassembled NF-H eluted at the void volume were used for observation.

Electron microscopy. Low-angle rotary shadowing was performed according to the method of Tyler and Branton (1980) as described previously (Hisanaga and Hirokawa, 1988). A small amount of NFs or the reassembled NF-H was diluted to adjust the protein concentration to about $100 \mu \mathrm{g} / \mathrm{ml}$ with $50 \%$ glycerol. Then, $50 \mu \mathrm{l}$ of the sample was sprayed onto a newly cleaved mica surface and, after drying under a vacuum at $5 \times 10^{-6}$ torr, rotary-shadowed with platinum at an angle of $6^{\circ}$ in Balzers BAF 301 .

QF-DE electron microscopy was carried out as described previously (Hirokawa and Heuser, 1981; Hisanaga and Hirokawa, 1987). The control and the dephosphorylated NFs were pelleted by centrifugation at $30,000 \times g$ for $30 \mathrm{~min}$. The precipitates were quick-frozen by contact with a pure copper block cooled by liquid helium. In some cases, to eliminate the artifacts by depositions of salt, the precipitates were fixed with 1\% glutaraldehyde, 2\% paraformaldehyde in PEM for $1 \mathrm{hr}$. After extensive washing with distilled water, they were quick-frozen as described above. The surface of the frozen samples were fractured at $-196^{\circ} \mathrm{C}$ at a vacuum of below $2 \times 10^{-6}$, etched for $8 \mathrm{~min}$ at $-95^{\circ} \mathrm{C}$, and replicated with platinum at an angle of $24^{\circ}$.

For thin-section electron microscopy, the control and the dephosphorylated NFs were precipitated by centrifugation at $30,000 \times g$ for 30 min and then fixed with $1 \%$ glutaraldehyde and $0.2 \%$ tannic acid in PEM. After being postfixed in cold $2 \% \mathrm{OsO}_{4}$ in $0.1 \mathrm{M}$ sodium cacodylate (pH 7.2), the samples were stained en bloc with $0.5 \%$ uranyl acetate for $2 \mathrm{hr}$. They were then dehydrated in ethanol and embedded in Epon 812. Thin sections were cut and double-stained with uranyl acetate and lead citrate.

Replicas and thin sections were viewed with a JEOL 1200EX electron microscope (JEOL, Tokyo, Japan) operating at $100 \mathrm{kV}$. Magnification was calibrated by photographs of phyrophyllite $(0.45 \mathrm{~nm})$.

Phosphate determination. The control and the dephosphorylated NFs or NF-H were ashed over a strong flame after an addition of $10 \%$ $\mathrm{Mg}\left(\mathrm{NO}_{3}\right)_{2} \cdot 6 \mathrm{H}_{2} \mathrm{O}$ in cthanol and solubilized in $1.2 \mathrm{~N} \mathrm{HCl}$. Pi reagent [a mixture of 1 vol of $10 \%(\mathrm{wt} / \mathrm{vol})$ ammonium molybdate and $3 \mathrm{vol}$ of $0.2 \%$ malachite green] was added to this solution. After $5 \mathrm{~min}$ the absorbance was measured at $660 \mathrm{~nm}$ (Stull and Buss, 1977).

Others. SDS-PAGE was performed according to Laemmli (1970). Protein concentrations were determined by the method of Bradford (1976) using BSA as a standard. Measurements of the lengths of the lateral projections and the core filaments were carried out with a Nexus 641 image processor (Nexus, Tokyo) as described previously (Hisanaga and Hirokawa, 1988).

\section{Results}

Structure of the projections of control and dephosphorylated NFS

To observe the projections by low-angle rotary shadowing, NFs should be prepared delicately. We first carried out the dephosphorylation with the NF fraction isolated as previously. However, it was difficult to obtain convincing results because of (1) a contamination by particles of alkaline phosphatase and (2) a low concentration of NFs after a rechromatography to remove alkaline phosphatase, although similar results to those described below had been obtained. We therefore chose to dephosphorylate NFs in the crude extract. The crude extract of the spinal cord was extensively dialyzed against a Tris-buffered solution to increase $\mathrm{pH}$ and to remove nucleotides and other dialysable substrate for phosphatase. Figure $1 A$ shows the time course of dephosphorylation. As already well known, NF-H and NF-M increased their electrophoretic mobility according to the extent of dephosphorylation. We used the mobility changes on SDSPAGE as the index of dephosphorylation. In this condition, dephosphorylation was almost accomplished in $5 \mathrm{hr}$. Further incubation did not increase the electrophoretic mobilities. NFs dephosphorylated by incubating with alkaline phosphatase for $5 \mathrm{hr}$ and the control NFs incubated without enzyme were isolated by Sepharose CL-4B, respectively. Gel filtration on Sepharose CL-4B alone was enough to enrich NF proteins for structural observation, although some contaminating proteins still remained in the fractions (Hisanaga and Hirokawa, 1988). Figure $1 B$ shows SDS-PAGE of control and dephosphorylated NFs, which were used for electron microscopic observation. 
Figure $2 a$ is a rotary-shadowed image of a NF isolated from the crude extract. As shown in the previous paper (Hisanaga and Hirokawa, 1988), many projections about 80-90 nm long extended from the core filament. Figure 2, $b, c$, shows the control and dephosphorylated NFs, respectively. Both the control and dephosphorylated NFs appeared knobby compared with the untreated filaments shown in Figure $2 a$. Incubation at $35^{\circ} \mathrm{C}$ alone seemed to make the core filaments knobby. Some of NFassociated proteins might bind to, or some of the projections might retract on, the core filament during incubation at $35^{\circ} \mathrm{C}$. Comparing control and dephosphorylated NFs, however, we did not detect structural differences in the projections. In both cases, the length of the projections was about $80-90 \mathrm{~nm}$ and the width about 4-5 $\mathrm{nm}$. The average length of the projections was $87 \pm 13 \mathrm{~nm}(n=119)$ for control NF and $82 \pm 12 \mathrm{~nm}(n$ $=142$ ) for dephosphorylated NF, respectively. With respect to the frequency, although it was difficult to estimate correctly, no distinct difference was detected, at least within our measurements. The number of projections per $22 \mathrm{~nm}$ along NFs were $4.5 \pm 0.5(n=7)$ for the control and $3.9 \pm 0.4(n=6)$ for the dephosphorylated NFs, respectively.

\section{Structure of the dephosphorylated filaments reassembled from purified $N F-H$}

The projections of the isolated NFs were composed of NF-H and NF-M (Hisanaga and Hirokawa, 1988). To investigate the effect of dephosphorylation more clearly, we next examined structural changes after dephosphorylation by using the filaments reconstructed from NF-H, which is the most phosphorylated subunit and the most probable candidate of the crossbridge. The time course of dephosphorylation of NF-H is shown in Figure $3 a$. Dephosphorylation was performed after the reconstruction of the filaments as described in Materials and Methods. The mobility of NF-H on SDS-PAGE increased according to dephosphorylation. A lower band in Figure $3 A$ is $E$. coli alkaline phosphatase. Figure $3 B$ shows the control $(a)$ and the dephosphorylated $(b)$ NF-H after gel filtration. These fractions were processed for rotary shadowing.

Rotary-shadowed images are shown in Figure 4. It is reported that long filaments are not formed from NF-H alone in the usual assembly condition (Geisler and Weber, 1981; Liem and Hutchison, 1982; Zackroff et al., 1982; Gardner et al., 1984). We also could not detect any long filaments having the same diameter as the isolated NFs. Instead, many short filaments, whose diameter was about $3 / 4$ of the native NFs, were reconstructed. Many projections-about $80 \mathrm{~nm}$ long and $4 \mathrm{~nm}$ wide-appeared from these short filaments (Fig. $4 a$ ). These sizes were almost the same as that of the native NFs. These projections seemed to correspond to the carboxy-terminal tail domains of NF-H. Even after dephosphorylation, the projections appeared as thin flexible structures extending from the short filaments (Fig. $4 b$ ). The dephosphorylated NF-H showed the projections with the same length and morphology as the control NFs. The average length of the projections was $75 \pm 11 \mathrm{~nm}(n=170)$ for the control and $78 \pm 12 \mathrm{~nm}(n=156)$ for the dephosphorylated filaments.

\section{Packing density of the control and the dephosphorylated NFs in the precipitates}

Rotary shadowing reveals the structure sprayed onto mica in the presence of a high concentration of glycerol. To eliminate possibilities that either the high concentration of glycerol or the adsorption to mica surface affects the structure of the projection,

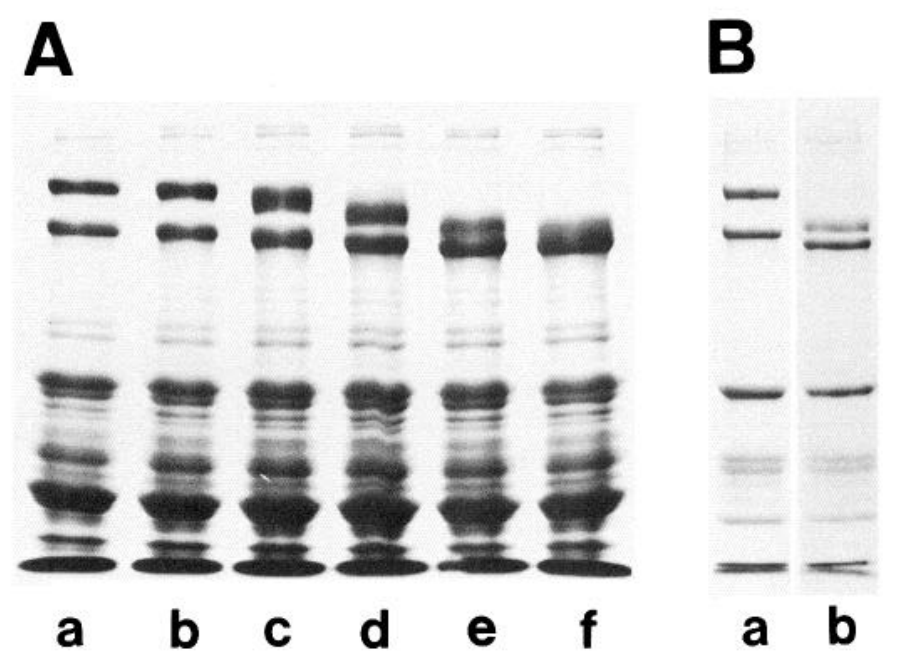

Figure 1. Dephosphorylation of NF proteins. A, 7.5\% SDS-PAGE of samples from a dephosphorylation time course experiment. Spinal cord extract $(80 \mu 1,10 \mathrm{mg} / \mathrm{ml})$ was incubated with $E$. coli alkaline phosphatase (4 units) at $35^{\circ} \mathrm{C}$ for $0 \mathrm{hr}$ (lane a), $0.5 \mathrm{hr}$ (lane b), $1 \mathrm{hr}$ (lane c), $2 \mathrm{hr}$ (lane d), $4 \mathrm{hr}$ (lane e), $7 \mathrm{hr}$ (lane $f$ ). Aliquots were withdrawn at the times indicated above, and dephosphorylation was terminated by boiling in a Laemmli (1970) buffer. B, 7.5\% SDS-PAGE of control (lane a) and dephosphorylated (lane $b$ ) NF after isolation through Sepharose CL-4B. Spinal cord extracts $(0.8 \mathrm{ml}, 10 \mathrm{mg} / \mathrm{ml})$ were incubated with and without 46 units of alkaline phosphatase at $35^{\circ} \mathrm{C}$ for $5 \mathrm{hr}$. At the end of incubation, they were applied on Sepharose CL-4B column and NFs were isolated.

we next studied the structure in a physiological solution. By using negative staining electron microscopy, Carden et al. (1985) have already reported that the ultrastructural appearance of the NFs was unaltered by the extensive dephosphorylation. Unfortunately, however, the projections that we would have liked to observe were not detected by negative staining. Therefore, we decided to examine the packing density of NFs in the precipitates by thin-section electron microscopy. With the same method, Kim et al. (1979) clearly showed the difference of density between microtubules with and without MAPs. In the case of NFs, however, the dephosphorylated NFs pelleted in the packing density similar to the control NFs (data not shown).

\section{Ability of the dephosphorylated projections of NFs to form cross-bridges}

Results described above indicated that the structure of the projections was not altered by dephosphorylation. Next was the problem of whether the dephosphorylated projections are able to form the cross-bridges between NFs. The cross-bridges can be detected most clearly by QF-DE electron microscopy. Freshly isolated NFs were precipitated and processed for QF-DE as described in Materials and Methods. Cross-bridges between the isolated NFs formed even in vitro (Fig. $5 a$ ). The length of these cross-bridges was $20-50 \mathrm{~nm}$ and the width, 5-10 $\mathrm{nm}$. These are the same sizes as observed in the axon. The frequency of the cross-bridges was not as abundant as in the axon. This may be because of the random orientation of NFs in the pellets. Although many proteins besides NF triplet proteins were still contaminated in the isolated NF fraction (Fig. $1 B$, lane a), the triplet proteins seemed to be sufficient to form the cross-bridges. The control NFs incubated without alkaline phosphatase are shown in Figure 5, $b, c$. The dephosphorylated NFs are shown in Figure $5, d-f$. The fields where NFs ran relatively parallel were selected 

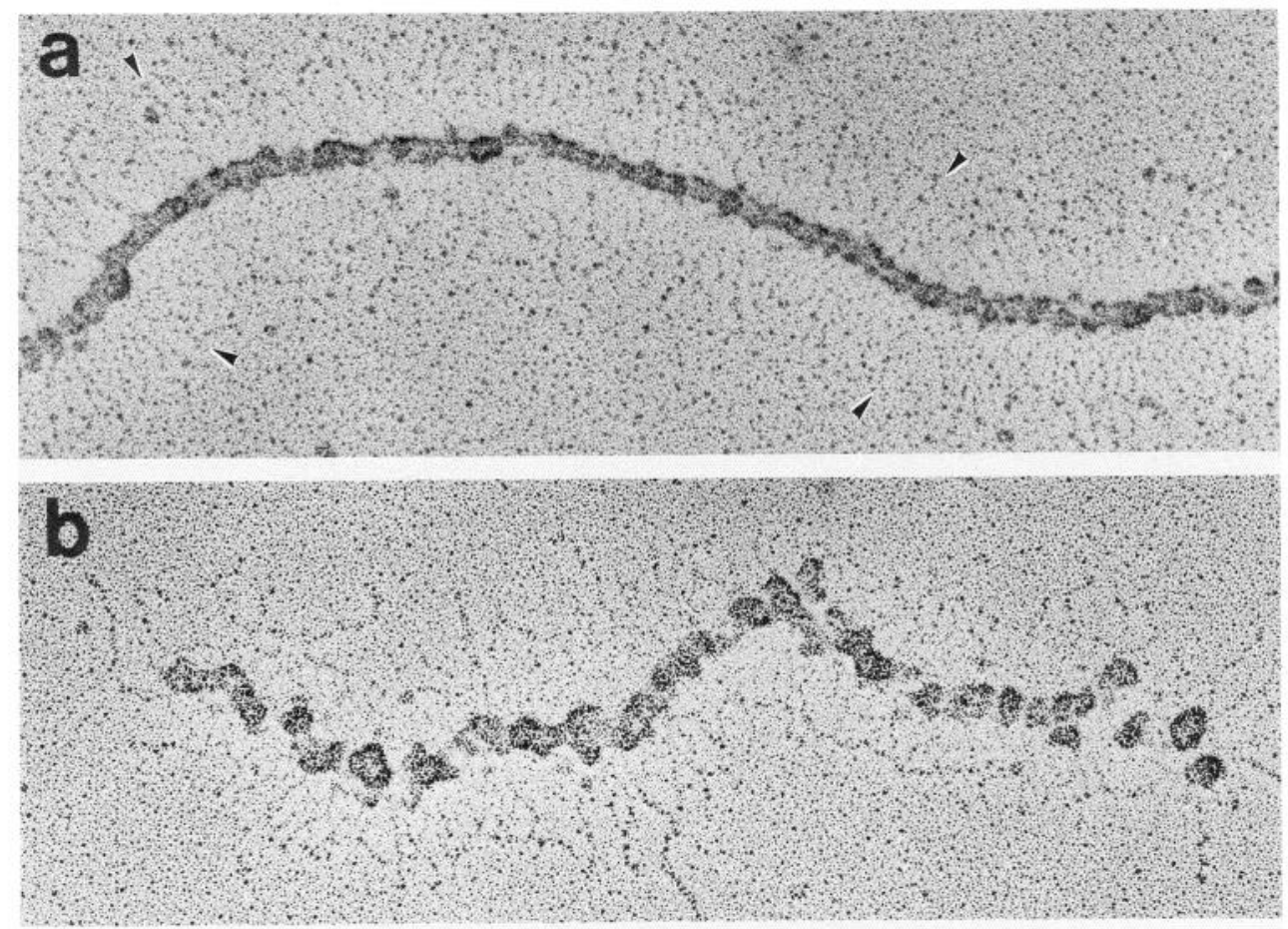

Figure 2. Low-angle rotary shadowing electron micrographs of the nontreated $(a)$, the control $(b)$, and the dephosphorylated $(c)$ NF. Numerous projections were observed extending laterally from the core filament. Arrowheads in $a$ indicate the backgroundprojections boundary. The core filament became knobby only after incubating at $35^{\circ} \mathrm{C}(b$ and $c)$. No distinct difference was observed in the structure of the projection between the control and the dephosphorylated NFs. Scale bar, $100 \mathrm{~nm}$.

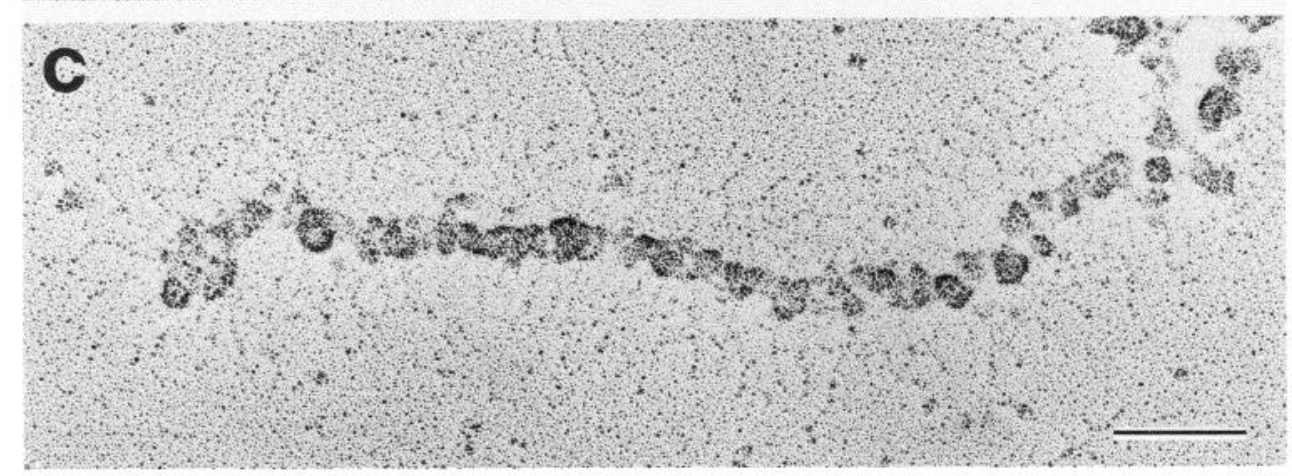

to show the cross-bridges clearly. The cross-bridges were observed between the dephosphorylated NFs, as well as between the control NFs. The frequency of the cross-bridges appeared to be similar between the control and the dephosphorylated NFs. Although the fixed samples are shown in Figure 5, the same results were obtained with freshly prepared ones.

\section{Determination of the phosphate content in the isolated $\mathrm{NF}$ and the short filament reconstructed from $\mathrm{NF}-\mathrm{H}$}

There is an inconsistency about the remaining phosphates after dephosphorylation. In order to determine the extent of dephosphorylation in this condition, we measured the phosphate content in isolated NF or NF-H before and after dephosphorylation. Although only about half the phosphate groups were removed in the isolated NF fraction, about $90 \%$ of the phosphate groups were removed from the short filaments reassembled from NF-H by incubating with $E$. coli alkaline phosphatase (Table 1). The relatively large amount of phosphate remaining after dephosphorylation in the isolated NF fraction may be due to contributions by contaminating phosphopolypeptides and phospholipids. The amount of the phosphate groups in NF-H subunit measured was about 2- to 3-fold larger than the values reported previously (Julien and Mushynski, 1982; Wong et al., 1984;
Carden et al., 1985). Assuming a molecular mass of $110 \mathrm{~K}$ for NF-H (Lees et al., 1988), however, the number of phosphate groups in NF-H would be $36 \mathrm{~mol} / \mathrm{mol} \mathrm{NF}-\mathrm{H}$; this value agrees relatively well with the number of putative phosphorylation sites $(>40)$, the common amino acid sequence motif (lysine-serineproline) (Geisler et al., 1987).

\section{Discussion}

One of the most interesting aspects of NFs is the phosphorylation. NFs display regionally different localization in neurons depending on their state of phosphorylation (Sternberger and Sternberger, 1982; Peng et al., 1986; Hart et al., 1987) and differential phosphorylation patterns during development (Dahl and Bignami, 1986; Dahl et al., 1986). Elucidation of the role of phosphorylation would lead to understanding the as yet unknown function of NF.

The effects of the phosphorylation of NF subunits have been thought to be mediated through the carboxy-terminal domains which are the multiple phosphate moieties in NF-M and NF$H$. The different organizations of NFs in axons and cell bodies/ dendrites suggested that phosphorylation particularly affects the structure of the cross-bridges. However, convincing results have not yet been obtained because the previous attempts to detect 
A
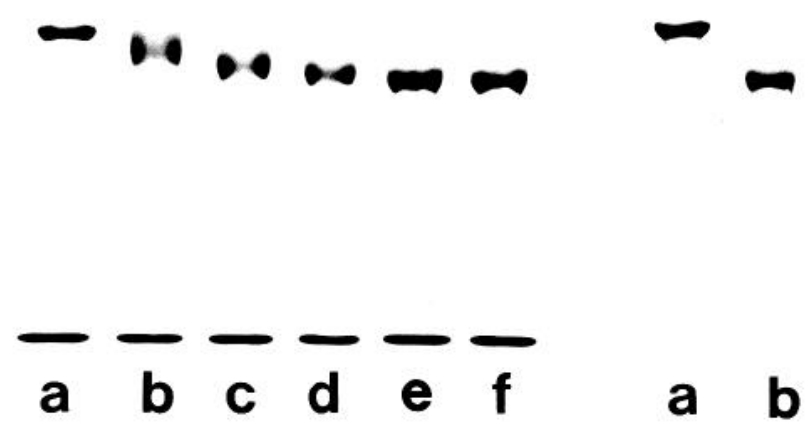

a $\mathbf{b}$

Figure 3. Dephosphorylation of NF-H. A, 7.5\% SDS-PAGE of samples from a dephosphorylation time course experiment. Short filaments reassembled from NF-H were dephosphorylated with alkaline phosphatase at $35^{\circ} \mathrm{C}$ for $0 \mathrm{hr}$ (lane a), $0.5 \mathrm{hr}$ (lane b), $1 \mathrm{hr}$ (lane c), $2 \mathrm{hr}$ (lane d), 4 $\mathrm{hr}$ (lane e), and $7 \mathrm{hr}$ (lane $f$ ). Aliquots were withdrawn at the times indicated above, and dephosphorylation was terminated by boiling in a Laemmli (1970) buffer. The lower band is $E$. coli alkaline phosphatase. $B, 7.5 \%$ SDS-PAGE of the control $(a)$ and the dephosphorylated $(b)$ NF-H after isolation through Sepharose CL-4B. Reassembled short filaments $(0.2 \mathrm{ml}, 1.8 \mathrm{mg} / \mathrm{ml})$ was incubated with and without 40 units of alkaline phosphatase at $35^{\circ} \mathrm{C}$ for $5 \mathrm{hr}$. At the end of incubation they were applied on Sepharose CL-4B gel filtration column, and NF-H eluted at void volume was electrophoresed.

the structural changes were mainly performed using negative staining (Carden et al., 1985; Georges et al., 1986), which is unsuitable for observing the structure of the projections. Our previous work indicated that low-angle rotary shadowing is the only method capable of revealing the detailed structure of the projection (Hisanaga and Hirokawa, 1988) and QF-DE is the best way to observe the cross-bridges (Hirokawa, 1982; Hirokawa et al., 1984).

Unexpectedly, however, dephosphorylation did not affect the structure of the projections, at least in vitro. This was ascertained with 2 samples: freshly isolated NFs and short filaments reas-
Table 1. Phosphate content of NF and NF-H

\begin{tabular}{|c|c|c|}
\hline & \multicolumn{2}{|c|}{$\begin{array}{l}\text { Phosphate content } \\
\text { (nmol phosphate/mg protein) }\end{array}$} \\
\hline & Control & Dephosphorylated \\
\hline \multirow[t]{3}{*}{ Isolated NF } & $290 \pm 27(n=4)$ & $148 \pm 26(n=4)$ \\
\hline & \multicolumn{2}{|c|}{$\begin{array}{l}\text { Phosphate content } \\
\text { (mol phosphate/mol polypeptide) }\end{array}$} \\
\hline & Control & Dephosphorylated \\
\hline NF-H & $\begin{array}{l}36 \pm 2 \quad(n=4)^{\prime} \\
65 \pm 4^{h}\end{array}$ & $\begin{array}{l}4 \pm 2 \quad(n=4)^{a} \\
8 \pm 5^{b}\end{array}$ \\
\hline
\end{tabular}

The phosphate contents before and after dephosphorylation were determined with the isolated NF and the filament reconstructed from NF-H. The numbers in parentheses represent the number of determinations. The listed values are means $\pm \mathrm{SD}$.

"The number of the phosphate groups of NF-H assuming molecular mass of 110 kDa (Lees et al., 1988).

"The number of the phosphate groups of NF-H assuming molecular mass of 200 $\mathrm{kDa}$.

sembled from NF-H. We think that these results are not artifacts caused by high concentrations of glycerol and/or adsorption onto mica surface. The structural change of myosin by phosphorylation has been demonstrated by the same method (Onishi and Wakabayashi, 1982; Craig et al., 1983). The packing density of NFs in the precipitates suggested that dephosphorylation did not change the structure of the projections even in the physiological solution, although the entire length of the projection was not observed in this case.

To study the effect of dephosphorylation on the projection of NF-H we used the short filament reassembled from NF-H. NF-H is incapable of forming long filaments in the absence of NF-L (Liem and Hutchison, 1982; Zackroff et al., 1982; Gardner et al., 1984). Negative staining experiments show that by dialyzing against the assembly solution, NF-H assembled into about $7 \mathrm{~nm}$ curly structures (Gardner et al., 1984), short filaments 8$15 \mathrm{~nm}$ in diameter and 30-150 $\mathrm{nm}$ long (Tokutake et al., 1984), and $15-30 \mathrm{~nm}$ spherical and $40-400 \mathrm{~nm}$ long aggregates (Moon et al., 1981). Our rotary shadowing images coincide generally with these observations. By dialyzing against the reassembly
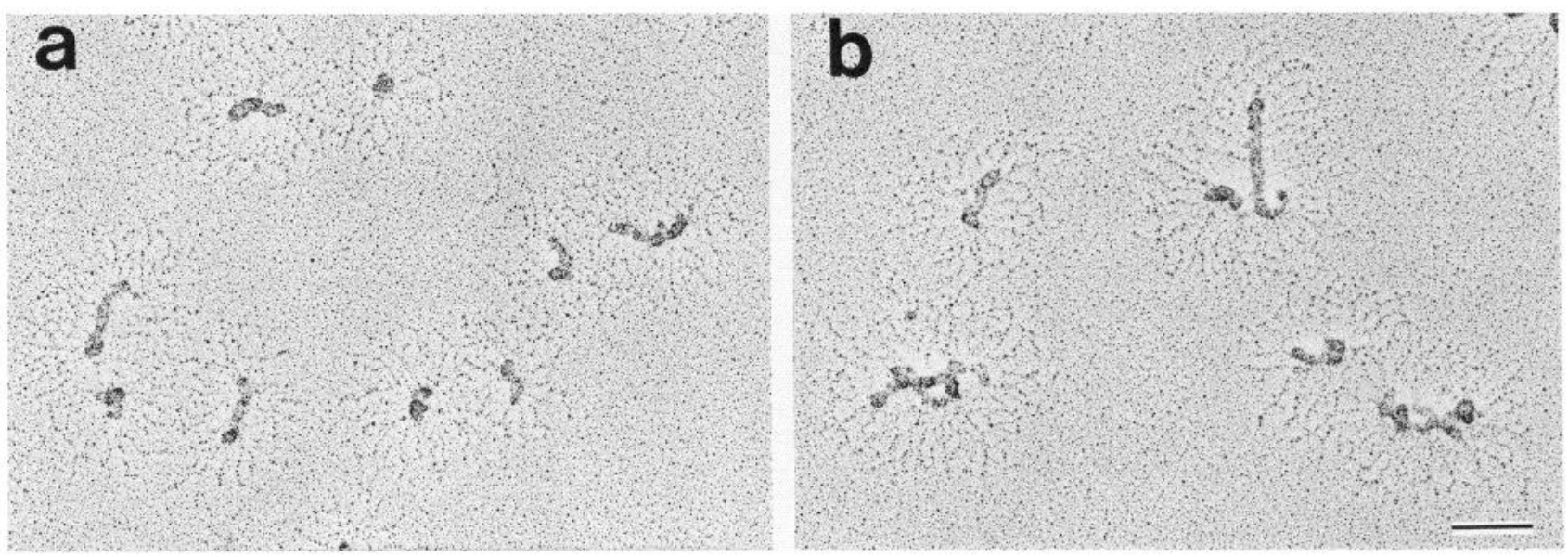

Figure 4. Low-angle rotary shadowing electron micrographs of the control (a) and the dephosphorylated $(b)$ NF-H short filaments. The short filaments assembled from NF-H were dephosphorylated, gel-filtered, and rotary-shadowed as described in Materials and Methods. Note that many projections appeared extending from the short filaments that were $60-360 \mathrm{~nm}$ long and $15 \mathrm{~nm}$ wide. Scale bar, $100 \mathrm{~nm}$. 

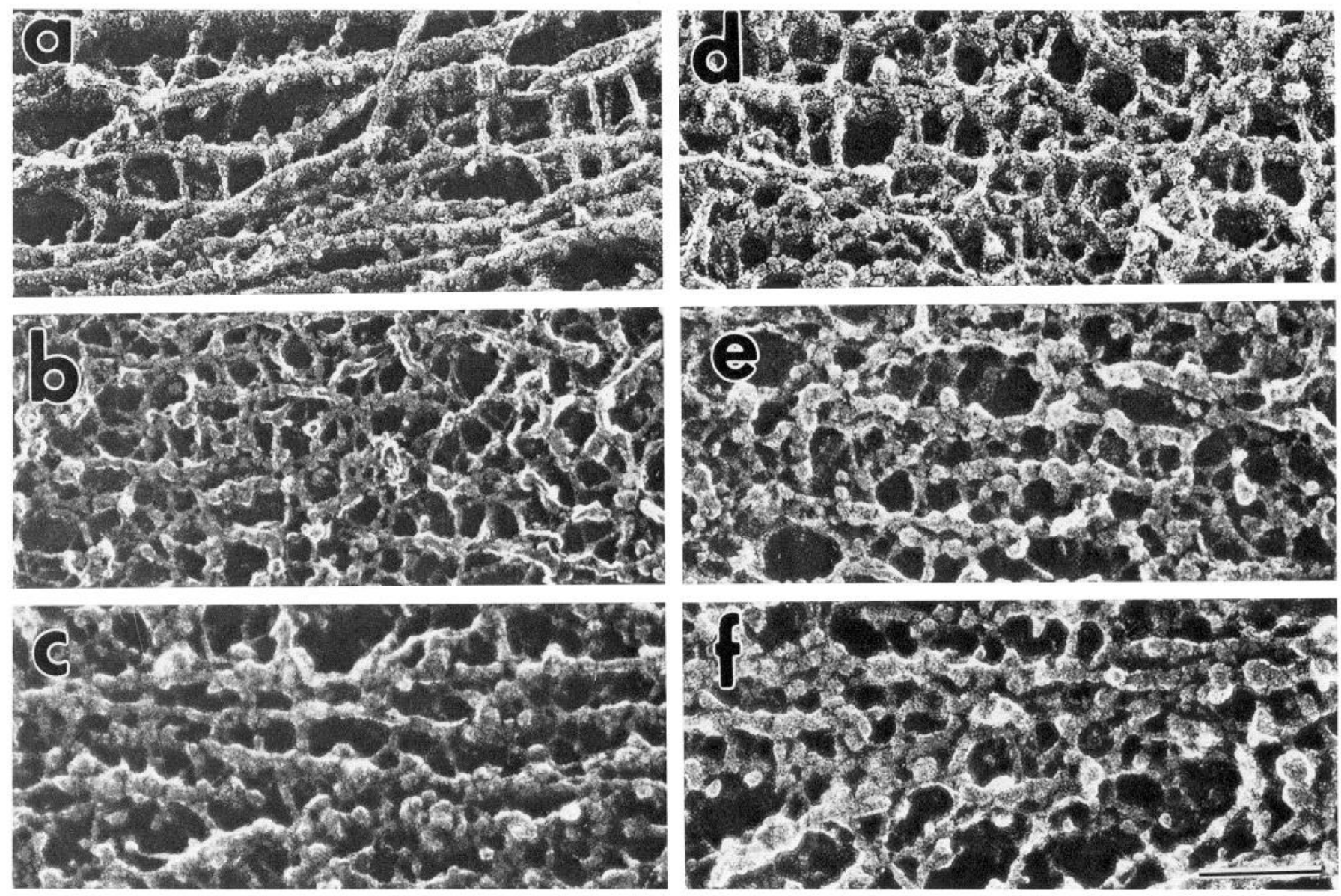

Figure 5. QF-DE electron micrographs of NFs. NFs isolated through Sepharose CL-4B were centrifuged at $30,000 \times g$ for 30 min at $4{ }^{\circ} \mathrm{C}$. The precipitates were processed for QF-DE as described in Materials and Methods. $a$, The NFs isolated directly from spinal cord extract without incubation. $b-c$, Control NFs incubated at $35^{\circ} \mathrm{C}$ for $5 \mathrm{hr}$ without enzyme; $d-f$, dephosphorylated NFs incubated with alkaline phosphatase at $35^{\circ} \mathrm{C}$ for $5 \mathrm{hr}$. Scale bar, $100 \mathrm{~nm}$.

buffer, NF-H formed short filaments having a rather uniform diameter of about $15 \mathrm{~nm}$. This value is about $3 / 4$ of that of isolated $(21 \mathrm{~nm})$ and reconstructed $(19 \mathrm{~nm})$ NFs (Hisanaga and Hirokawa, 1988). The large diameters are due to both decoration by glycerol and the thickness of the replica. Assuming the real width of NF to be $10 \mathrm{~nm}, 3 / 4$ of this value is $7.5 \mathrm{~nm}$, which agrees well with the $7 \mathrm{~nm}$ reported by Gardner et al. (1984). In this case, too, the $\alpha$-helix-rich rod domain seems to contribute to form the short filaments and the carboxy-terminal tail domain seems to constitute the projections as well in the intact NFs.

The cross-bridges were formed in vitro between NFs. The cross-bridges were observed with both fresh and fixed NFs. This eliminates the artifact by the deposition of salt. The sizes were very similar to those observed in vivo. Cross-bridges in both control and dephosphorylated NFs were about $15-60 \mathrm{~nm}$ long and $5-10 \mathrm{~nm}$ wide. These cross-bridges seemed to be comprised of the carboxy-terminal tail domain of NF-H and NF-M. This is supported by the fact that such apparent cross-bridges were not observed between filaments reconstructed from NF-L alone (Hirokawa et al., 1988). Whether a single projection can form a cross-bridge is unknown. That the cross-bridges were thicker than the projections may suggest a possibility that a single crossbridge is composed of several projections. Although we think that NF triplet polypeptides are sufficient to form the crossbridges, a possibility that proteins other than NF-M and NF-H are required to form cross-bridge cannot be excluded on the basis of our experiments because many proteins besides triplet proteins were present in the NF fraction.

There are some contradicting reports about the phosphates that remained after the dephosphorylation by $E$. coli alkaline phosphatase. Carden et al. (1985) reported that $E$. coli alkaline phosphatase remove $>90 \%$ of the phosphate groups from serine residue of NF-M and NF-H. In contrast, Georges et al. (1986) observed that bovine NF dephosphorylated under a similar condition still contained a considerable amount of phosphate (about a third of untreated NF). Our result - that most of the phosphate groups of NF-H were removed by the treatment with alkaline phosphatase-coincides with the result of Carden et al. (1985). Considering our data of the phosphate measurement, the results of structural observations described above can be put in the following way: Removal of most phosphate groups from NF-H did not affect the structure of the projections, at least, of NF-H. Assuming the molecular mass of NF-H to be $200 \mathrm{kDa}$, so as to facilitate comparison of our data with those of previous studies, the number of the phosphate groups in NF-H would be $65 \mathrm{~mol}$ phosphate/mol NF-H (Table 1), which is in between those (about 20-30 mol/mol NF-H) of Julien and Mushynski (1982), Wong et al. (1984), Carden et al. (1985), and Georges et al. (1986), and that $(100 \mathrm{~mol} / \mathrm{mol} \mathrm{NF}-\mathrm{H})$ of Jones and Williams (1982). Taking the molecular mass of NF-H to be $110 \mathrm{kDa}$, as recently 
estimated from the DNA sequence of NF-H (Lees et al., 1988), $\mathrm{NF}-\mathrm{H}$ would contain $36 \mathrm{~mol}$ phosphate/mol polypeptide, which agrees relatively well with the number of the common motif of phosphorylation sites in NF-H (Geisler et al., 1987; Lees et al., 1988).

The present study indicates that dephosphorylation does not affect the structure of the projections, although it is possible that we overlooked small conformational changes. This brings us back to the initial question of why the organizations of NFs in the cell bodies and the dendrites differ from those in the axons. We think that there are 3 possible explanations. The first is simply the density of NFs. The number of NFs in the cell bodies and the dendrites is less than in the axons. Affinity may not be strong enough to form the cross-bridges between dispersed, randomly oriented NFs. The cross-bridges might be formed only after NFs enter the axon, where the concentration of NF might be sufficiently high for the projections to interact with the neighboring NFs. The second is that the dephosphorylated projections are bound by some NF-associated proteins that inhibit formation of cross-bridges within the cell bodies. Otherwise, some NF-associated protein(s), which localize mainly in the axon, may be required to form cross-bridges. The third is the content of NF-H and NF-M in the NFs. There are several different reports on the molar ratio of NF-L, NF-M, and NF-H in the NF fraction (Shecket and Lasek, 1980; Scott et al., 1985). One of the reasons for this discrepancy may be due to differences in starting materials for preparation of NFs, e.g., white matter-rich fraction vs gray matter-rich fraction.

Although the dephosphorylation at the carboxy-terminal tail domain of NF-M and NF-H did not change the structure of NFs at least in vitro, we think the phosphorylation may still play important roles in the function of NFs, such as regulation of the interactions of NFs with other cytoplasmic organelles and/or proteins. Further studies are necessary to understand the functional significance of NF phosphorylation.

\section{References}

Bennett, G. S., and C. Dilullo (1985) Slow posttranslational modification of a neurofilament protein. J. Cell Biol. 100: 1799-1804.

Bradford, M. M. (1976) A rapid and sensitive method for the quantitation of microgram quantities of protein utilizing the principle of protein-dye binding. Anal. Biochem. 72: 248-254.

Carden, M. J., W. W. Schlaepfer, and V. M.-Y. Lee (1985) The structure, biochemical properties, and immunogenicity of neurofilament peripheral regions are determined by phosphorylation state. J. Biol. Chem. 260: 9805-9817.

Chin, T. K., P. A. M. Eagles, and A. Maggs (1983) The proteolytic digestion of ox neurofilaments with trypsin and $\alpha$-chymotrypsin. Biochem. J. 215: 239-252.

Cohen, R. S., H. C. Pant, S. House, and H. Gainer (1987) Biochemical and immunocytochemical characterization and distribution of phosphorylated and nonphosphorylated subunits of neurofilaments in squid giant axon and stellate ganglion. J. Neurosci. 7: 2056-2074.

Craig, R., R. Smith, and J. Kendrick-Jones (1983) Light-chain phosphorylation controls the conformation of vertebrate nonmuscle and smooth muscle myosin molecules. Nature 302: 436-439.

Dahl, D., and A. Bignami (1986) Neurofilament phosphorylation in development. Exp. Cell Res. 162: 220-230.

Dahl, D., C. J. Crosby, E. E. Gardner, and A. Bignami (1986) Delayed phosphorylation of the largest neurofilament protein in rat optic nerve development. J. Neurosci. Res. 15: 513-519.

Gardner, E. E., D. Dahl, and A. Bignami (1984) Formation of 10namometer filaments from the $150 \mathrm{~K}$-dalton neurofilament protein in vitro. J. Neurosci. Res. 11: 145-155.

Geisler, N., and K. Weber (1981) Self-assembly in vitro of the 68,000 molecular weight component of the mammalian neurofilament triplet proteins into intermediate-sized filaments. J. Mol. Biol. 151: 565571.

Geisler, N., E. Kaufmann, S. Fischer, U. Plessmann, and K. Weber (1983) Neurofilament architecture combines structural principles of intermediate filaments with carboxy-terminal extensions increasing in size between triplet proteins. EMBO J. 8: 1295-1302.

Geisler, N., S. Fischer, J. Vandekerckhove, J. Van Damme, U. Plessmann, and K. Weber (1985) Protein-chemical characterization of $\mathrm{NF}-\mathrm{H}$, the largest mammalian neurofilament component; intermediate filament-type sequences followed by a unique carboxy-terminal extension. EMBO J. 4: 57-63.

Geisler, N., J. Vandekerckhove, and K. Weber (1987) Localization and sequence characterization of the major phosphorylation sites of the high molecular mass neurofilament proteins $M$ and $H$. FEBS Lett. 221: 403-407.

Georges, E., S. Lefebvre, and W. E. Mushynski (1986) Dephosphorylation of neurofilaments by exogenous phosphatases has no effect on reassembly of subunits. J. Ncurochem. 47: 477-483.

Glicksman, M. A., D. Soppet, and M. B. Willard (1987) Posttranslational modification of neurofilament polypeptides in rabbit retina. J. Neurobiol. 18: 167-196.

Hart, C. E., G. H. Nuckolls, and J. G. Wood (1987) Subcellular compartmentalization of phosphorylated neurofilament polypeptides in neurons. Cell Motil. Cytoskel. 7: 393-403.

Hirokawa, N. (1982) Cross-linker system between neurofilaments, microtubules, and membranous organelles in frog axons revealed by the quick-freeze, deep-etching method. J. Cell Biol. 94: 129-142.

Hirokawa, N., and J. E. Heuser (1981) Quick-freeze, deep-etch visualization of the cytoskeleton beneath surface differentiations of intestinal epithelial cells. J. Cell Biol. 91: 399-409.

Hirokawa, N., M. A. Glicksman, and M. B. Willard (1984) Organization of mammalian neurofilament polypeptides within the neuronal cytoskeleton. J. Cell Biol. 98: 1523-1536.

Hirokawa, N., S. Hisanaga, and Y. Shiomura (1988) MAP2 is a component of crossbridges between microtubules and neurofilaments in the neuronal cytoskeleton: Quick-freeze, deep-etch immunoelectron microscopy and reconstitution studies. J. Neurosci. 8: 2769-2779.

Hisanaga, S., and N. Hirokawa (1987) Substructure of sea urchin egg cytoplasmic dynein. J. Mol. Biol. 195: 919-927.

Hisanaga, S., and N. Hirokawa (1988) Structure of the peripheral domains of neurofilaments revealed by low angle rotary shadowing. J. Mol. Biol. 202: 297-305.

Jones, S. M., and R. C. Williams, Jr. (1982) Phosphate content of mammalian neurofilaments. J. Biol. Chem. 257: 9902-9905.

Julien, J.-P., and W. E. Mushynski (1982) Multiple phosphorylation sites in mammalian neurofilament polypeptides. J. Biol. Chem. 257: 10467-10470.

Julien, J.-P., and W. E. Mushynski (1983) The distribution of phosphorylation sites among identified proteolytic fragments of mammalian neurofilaments. J. Biol. Chem. 258: 4019-4025.

Kim, H., L. I. Binder, and J. L. Rosenbaum (1979) The periodic association of MAP2 with brain microtubules in vitro. J. Cell Biol. 80: 266-276.

Laemmli, U. K. (1970) Cleavage of structural proteins during the assembly of the head of bacteriophage T4. Nature 227: 680-685.

Lee, V. M.-Y., M. J. Carden, W. W. Schlaepfer, and J. Q. Trojanowski (1987) Monoclonal antibodies distinguish several differentially phosphorylated states of the two largest rat neurofilament subunits (NF-H and NF-M) and demonstrate their existence in the normal nervous system of adult rats. J. Neurosci. 7: 3474-3488.

Lees, J. F., P. S. Shneidman, S. F. Skuntz, M. J. Carden, and R. A. Lazzarini (1988) The structure and organization of the human heavy neurofilament subunit (NF-H) and the gene encoding it. EMBO J. 7 : 1947-1955.

Liem, R. K. H., and S. B. Hutchison (1982) Purification of individual components of the neurofilament triplet: Filament assembly from the 70,000-dalton subunit. Biochemistry $21: 3221-3226$.

Liem, R. K. H., S. S. M. Chin, E. Moraru, and E. Wang (1985) Monoclonal antibodies to epitopes on different regions of the 200,000 dalton neurofilament protein. Exp. Cell Res. 156: 419-428.

Minami, Y., and H. Sakai (1985) Dephosphorylation suppresses the activity of neurofilament to promote tubulin polymerization. FEBS Lett. 185: 239-242.

Moon, H. M., T. Wisniewski, P. Merz, J. De Martini, and H. M. Wisniewski (1981) Partial purification of neurofilament subunits from 
bovine brains and studies on neurofilament assembly. J. Cell Biol. 89: 560-567.

Myers, M. W., R. A. Lazzarini, V. M.-Y. Lee, W. W. Schlaepfer, and D. L. Nelson (1987) The human mid-size neurofilament subunit: A repeated protein sequence and the relationship of its gene in the intermediate filament gene family. EMBO J. 6: 1617-1626.

Oblinger, M. M. (1987) Characterization of posttranslational processing of the mammalian high-molecular-weight neurofilament protein in vivo. J. Neurosci. $7: 2510-2521$.

Onishi, H., and T. Wakabayashi (1982) Electron microscopic studies of myosin molecules from chicken gizzard muscle. I: The formation of the intramolecular loop in the myosin tail. J. Biochem. 92: 871879.

Peng, I., L. I. Binder, and M. M. Black (1986) Biochemical and immunological analyses of cytoskeletal domains of neurons. J. Cell Biol. 102: $252-262$

Scott, D., K. E. Smith, B. J. O'Brien, and K. J. Angelides (1985) Characterization of mammalian neurofilament triplet proteins. J. Biol. Chem. 260: 10736-10747.

Sharp, G. A., G. Shaw, and K. Weber (1982) Immunoelectron microscopical localization of the three neurofilament triplet proteins along neurofilaments of cultured dorsal root ganglion neurons. Exp. Cell Res. 137: 403-413.
Shecket, G., and R. J. Lasek (1980) Preparation of neurofilament protein from guinea pig peripheral nerve and spinal cord. J. Neurochem. 35: 1335-1344.

Sternberger, L. A., and N. H. Sternberger (1983) Monoclonal antibodies distinguish phosphorylated and nonphosphorylated forms of neurofilaments in situ. Proc. Natl. Acad. Sci. USA 80: 6162-6170.

Stull, J. T., and J. E. Buss (1977) Phosphorylation of cardiac troponin by cyclic adenosine $3^{\prime}: 5^{\prime}$-monophosphate-dependent protein kinase. J. Biol. Chem. 252: 851-857.

Tokutake, S., R. K. H. Liem, and M. L. Shelanski (1984) Each component of neurofilament assembles itself to make component-specific filament. Biomed. Res. 5: 235-238.

Tyler, J. M., and D. Branton (1980) Rotary shadowing of extended molecules dried from glycerol. J. Ultrastruct. Res. 71: 95-102.

Willard, M., and C. Simon (1981) Antibody decoration of neurofilaments. J. Cell Biol. 89: 198-205.

Wong, J., S. B. Hutchison, and R. K. H. Liem (1984) An isoelectric variant of the 150,000-dalton neurofilament polypeptide. J. Biol. Chem. 259: 10867-10874.

Zackroff, R. V., W. W. Idler, P. M. Steinert, and R. D. Goldman (1982) In vitro reconstitution of intermediate filaments from mammalian neurofilament triplet polypeptides. Proc. Natl. Acad. Sci. USA 79: 754-757. 